\title{
Case Study: Telemedicine for Arrhythmia Care: Early Detection of Co-Existing Conditions
}

\author{
Kathleen Fasing* \\ DNP, MS, ACNP Madonna University, Livonia MI, University of Michigan, Staff ACNP, Michigan, USA
}

${ }^{\star}$ Corresponding author: Kathleen Fasing DNP, MS, ACNP Madonna University, Livonia MI, University of Michigan, Staff ACNP, Ann Arbor, Michigan, USA, Phone: (734)232-1692; Fax: (734)232-6538

Received: December 22, 2020; Accepted: December 28, 2020; Published: January 04, 2020

After two years of dedication to the research study Telemedicine: Enabling Patients in Self-Care Behaviors (TEPSCB) I am continually pleased with the phenomenon of detecting co-existing diseases much earlier than with conventional approaches to patient visits. Identifying co-existing diseases is not an end point of the study but is a benefit which has been observed with several individuals in the Telemedicine group. Historically, the arrhythmia department within our local Midwest tertiary care facility has seen patients on a six month or yearly return visit basis. These appointments have been arranged as an in person visit and have entailed an office visit with labs, electrocardiogram (ECG), and follow up phone calls to answer any post visit questions. Currently, with the TEPSCB study, 30 patients are enrolled in the telemedicine group having telemedicine video visits occurring once monthly for three months. Another 30 patients are enrolled in the conventional six-month in person visit group. With more frequent and readily accessible video visits it has become very easy to diagnose and treat co-morbid conditions which can otherwise exacerbate arrhythmias.

The Telemedicine: Enabling Patients in Self-Care Behaviors study has demonstrated several instances in which diabetes mellitus, hypertension, and other conditions can be identified within a few short months and thus the patient receives care much quicker than with conventional six-month visits. This case study will enlighten the ease with which earlier video visits enable ongoing feedback, symptom reporting, and earlier response from our health care team. To fully understand the case study, please allow some background on the Telemedicine: Enabling Patients in Self-Care Behaviors. The following is the theory behind the study and the inspiration for the study. It becomes important to give the background of the initiation of the Telemedicine: Enabling Patients in Self-Care Behaviors.

The first outcome of the study is to determine if patients enrolled in a telemedicine program for the care of cardiac arrhythmias have any difference in [1] time of arrhythmia recognition, [2] time of arrhythmia diagnosis by a healthcare provider, and [3] time of treatment initiation compared with patients enrolled in standard care for cardiac arrhythmias. In addition, this study examines patient's self-efficacy related to medication use, functional self-efficacy, and perceived arrhythmia symptoms. The second outcome of the telemedicine arrhythmia pilot study includes a theme noted in the Integrated Theory of Health Behavior Change (ITHBC) which assists the individual in increasing one's involvement in one's own healthcare via a process of increased social support, increased self-awareness, and increased selfefficacy (Ryan, P., 2009). This theory relates directly to the arrhythmia population in its focus upon the patient's self-awareness, increased social support (promoted with the frequent telemedicine visits and teaching for patients and family), and increased self-efficacy and understanding of one's arrhythmia and management of one's arrhythmia. The intent of this outcome is to assist, enable, and educate the patient in managing day to day changes in one's arrhythmia symptoms. This process is aimed at increasing one's self-efficacy in coping with the arrhythmia and increasing one's ability to react to arrhythmia changes. The second outcome is measured with the MUSE, FSES and the ASTA surveys which measure participants medication use self-efficacy, functional status self-efficacy, and arrhythmia self-efficacy. The surveys are given to all participants at the beginning and end of the study.

This type of telemedicine program is based upon several studies which have shown improved clinical outcomes with the use of telemedicine. One such telemedicine study includes those with an Implantable Cardioverter Defibrillator (ICD). The TRUST trial compares the use of a telephone video conference to conventional in person follow up visits. The TRUST trial determined the efficacy and safety for monitoring ICDs and the reduction of in person follow up appointments (Dalouk, K. et. al., 2017; Varma, N. et.al, 2010). This telemedicine trial was a retrospective trial looking at the time to first appropriate ICD therapy (device shocking in the presence of a dangerous arrhythmia) and the time to first inappropriate ICD therapy (device shocking in the presence of a non-dangerous arrhythmia) (Dalouk, K. et.al., 2017). The TRUST trial compared the safety and usefulness of remote monitoring in ICD recipients and conventional in person ICD follow up visits. Endpoints respective to the study included non-inferior outcomes for telemedicine in those who had no care visit available near their homes with in-person visits. There was no difference between home monitoring and those with conventional appointments with adverse event rates of 10.4 for each group. (Dalouk, K., et al., 2017; Varma, N. et. al., 2013).

This telemedicine arrhythmia pilot study has tried to mimic the early detection data of the TRUST trial. An outcome of the telemedicine arrhythmia project is quality improvement to gain a timely recognition, diagnosis, and prompt treatment of abnormal arrhythmias. Through this early detection, treatment may entail 
a change in the AAD drug, a change in the dose of the drug, or an adjustment to the heart rate parameters and rhythm programing of a device. In addition, recognition of the need for a procedure which may eliminate the source of the arrhythmia via radiofrequency ablation (Lee, H-C., Huang, K., and Shen, W-K., 2011). Needed treatment may be quite simplistic as a minor change in dosing of medication, eliminating the medication, changing to another medication, or making subtle or large changes in the pacemaker or ICD programming, which may eliminate the arrhythmia (Varma, N. et.al, 2015).

Utilizing the study to determine if there is any difference in the time of recognition, diagnosing and treatment of any new arrhythmia with individuals in the Telemedicine versus the Standard visit group has already shown an improved time to diagnosis and treatment in the telemedicine group. The final statistics are not determined as this is an ongoing study. All 60 participants have been randomized and over $1 / 3$ of the participants have already completed the study. The initial overall consensus is the telemedicine group simply due to the frequent follow up visits and the use of monitors such as the Kardia, loop recorders, pacemakers and internal cardioverter defibrillators (ICDs) has a clear advantage and tendency toward improved time to recognize, diagnose and treat the arrhythmia.

The case study involves a young woman who enrolled in the study and was randomized to the telemedicine group. She is a woman who is 49 years old with a distant history of a premature ventricular contraction (PVC) with a PVC ablation in 2013 and episodes of paroxysmal supraventricular tachycardia. She was feeling well for many years and over the last one year has ongoing palpitations, chest pain and fatigue which became disabling. She had a normal left ventricular ejection fraction (EF) of $60 \%$ in 2018, which became worse over the span of two years. Her chief complaint remained strong and painful palpitations, chest pain with activity and fatigue. Over several months her complaints continued to include symptoms of chest pressure, fatigue, and activity intolerance.

Her event monitors continued to show 1-2\% PVCs, short episodes of SVT with an SVT burden of 2-3\%. She underwent a treadmill stress test and nuclear medicine stress test which were both negative for ischemic heart disease. She had been seen in our office every six months for follow up appointments, prior to enrolling in the TEPSCB. She began to feel much worse and felt her symptoms were completely related to an arrhythmia etiology, despite her relatively negative event monitors. She was very symptomatic and always related her chest pain, fatigue, and palpitations to an arrhythmia. She enrolled in the Telemedicine study and was randomized to the telemedicine group. Once in the study she had a series of monthly appointments via Zoom ${ }^{\mathrm{T}}$ meetings in which her symptoms, possible work up strategies and options were discussed. Via these frequent and concentrated video visits she underwent updated MRIs and PET scans which led to the question if she had sarcoidosis of the heart.

\section{The following include results of her studies:}

Hx of a Biallelic mutation of HFE2 gene which has been seen with hemochromatosis. Further testing showed the mutation however was not consistent with iron overload of the heart.

\section{Gene RX: Hereditary Hemochromatosis}

\section{Cardiac MRI March 2020}

No late gadolinium enhancement. LV Ejection Fraction (EF) 45\%, RV (EF) 30\%. Note of RV EF decreased from a prior study showing $48 \%$ the year prior.

\section{Cardiac MRI July 2020}

Late gadolinium enhancement at the mid ventricular level involving the inferior septum and the inferior wall in a non-ischemic distribution. LV Ejection Fraction (EF) 47\% and RV (EF) 46\%.

\section{PET Scan: June 2020}

\section{Nuclear Medicine PET}

LV perfusion is noted as normal. There is increased FDG myocardial uptake most intense in the mid inferolateral wall, but also including at a lower intensity the inferior, inferoseptal and anterolateral

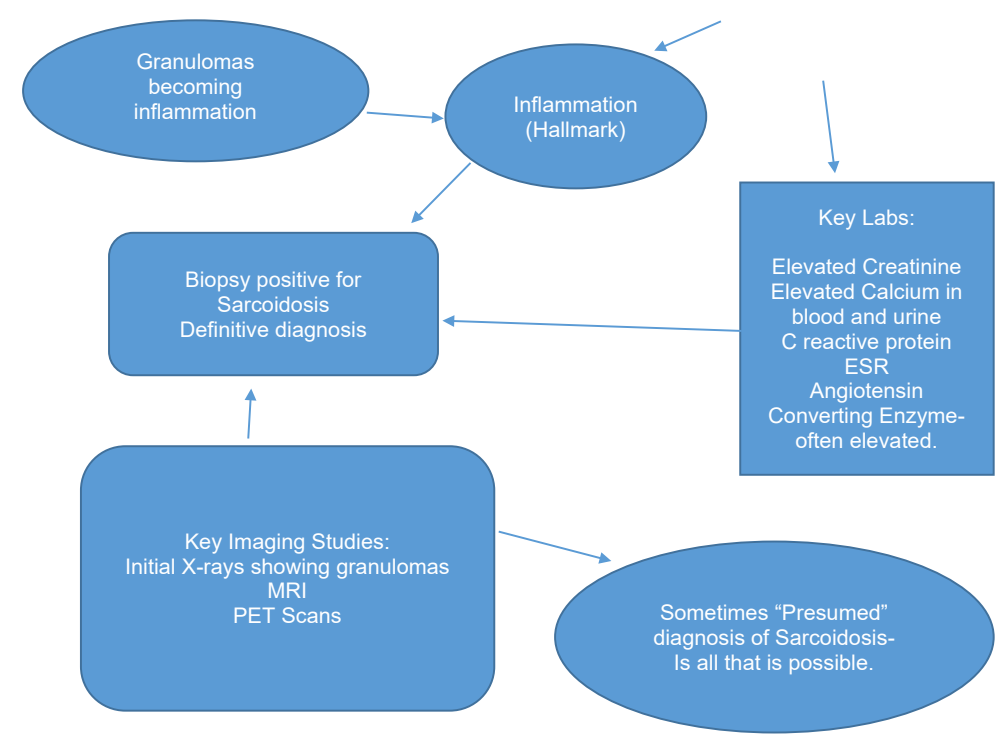

Chart for Helpful Work Up of Sarcoidosis 
segments consistent with myocardial inflammation.

The PET scan showed increased FDG. The Triponin was negative There was no other evidence of inflammation and no evidence of Sarcoidosis.

\section{Zio Patch}

$8 / 20205$ beats of nun-sustained ventricular tachycardia and an 8 beat run of supraventricular tachycardia.

\section{Echocardiogram}

Showing variable decreased LV ejection fraction.

The patient work-up was expeditiously arranged and with her gene mutation, iron deposits within the heart were suspected, but were not present. There was also concern for the possibility of sarcoidosis, but the PET scan, negative troponin and no other inflammation eliminated this possibility. She was evaluated with the genetics department, structural heart disease specialists and the heart failure group. She was diagnosed with heart failure and her diuretic dosing and angiotensin converting enzyme were increased and she underwent physical therapy to assist in improving symptoms of heart failure. Within 3-4 months her symptoms improved greatly.

This case study is an example of a very expeditious evaluation of symptoms of chest pressure, fatigue, and activity intolerance The TEPSCB assisted in meeting regularly with this young lady and expedited video appointments to update symptoms, discuss ongoing testing, and refer her to a myriad of specialist to evaluate for suspected myocardial inflammation and determine a treatment plan. The diagnosis of sarcoidosis was thankfully negative. The telemedicine study allowed a very timely evaluation, prompt diagnosis of heart failure and improved treatment. This case study shows, although not a main endpoint of the
Telemedicine: Enabling Patients in Self-Care Behaviors (TEPSCB) study, that prompt diagnosis of a coexisting condition is greatly expedited with the use of telemedicine and video visits.

There are many concurrent diagnosis' which can occur with arrhythmias and it often takes months to years to evaluate such concurrent diagnosis, as office visits, work up and chief complaints progress through several changes over time. A telemedicine evaluation can speed the process of the assessment of these items and expedite prompt care. In the case of a sarcoidosis work up, key elements of sarcoidosis are either confirmed or eliminated. This example case has failed to show these elements and the required inflammation needed for a sarcoidosis diagnosis and is only positive for a heart failure diagnosis.

Keywords: Telemedicine, Arrhythmia, Co-existing, Early detection

\section{References}

1. Dalouk K, Gandhi N, Jessel P, MacMurdy K, Zarraga I, et al. (2017) Outcomes of telemedicine video-conferencing clinic versus in-person clinic follow-up for implantable card'ioverter-defibrillator recipients, Circulation Arrhythmia Electrophysiology 10.

2. Lee, H-C, Huang K, Shen, W-K (2011) Use of antiarrhythmic drugs in elderly patients, Journal of Geriatric Cardiology 8 (3): 184-194. [crossref]

3. Ryan P (2009) Integrated theory of health behavior change: Background and intervention development, Clinical Nurse Specialist 23 (3): 161-172. [crossref]

4. Varma N, Epstein A, Irimpen A, Schweikert R, Love C (2010) Efficacy and safety of automatic remote monitoring for implantable cardioverter-defibrillator follow-up: The Lumos-T safely reduces routine office device follow-up, TRUST trial, Circulation 122: 325-332. [crossref]

5. Varma N, Ricci, R (2013) Telemedicine and cardiac implants: what is the benefit? European heart journal 34 (25), 1885-1895.

6. Varma N, Ricci, R (2015) Impact of remote monitoring on clinical outcomes, Journal of Cardiovascular Electrophysiology 25, (12). 